\title{
COMMENTARY
}

\section{Lacrimal Gland Postganglionic Innervation}

\section{Unveiling the Role of Parasympathetic and Sympathetic Nerves in Stimulating Tear Secretion}

\author{
Karina Dias-Teixeira
}

From the Department of Cell Biology, University of Virginia, Charlottesville, Virginia

The tear film is a thin fluid layer comprising over 1800 different extracellular proteins and many diverse species of lipids ${ }^{1}$ that are protective and important in the homeostasis of the avascular cornea as an interface between the ocular surface and the environment. ${ }^{2}$ Tear deficiency is associated with loss of homeostasis, giving rise to dry eye syndrome, a disease affecting $5 \%$ to $7 \%$ of the world's population. ${ }^{3}$ As the main source of basal and reflex tears, the lacrimal gland plays a critical role in maintaining ocular surface homeostasis. To better understand dry eye and how lacrimal gland autonomic innervation controls tearing, Jin et $\mathrm{al}^{4}$ studied lacrimal gland innervation in a mouse model.

\section{Identification of the Postganglionic Nerves Projecting to the Lacrimal Gland}

In this issue of The American Journal of Pathology, Jin et $\mathrm{al}^{4}$ identify the distribution and routes of postganglionic nerves using a different and more precise approach than previously used. Lacrimal gland innervation is by both sympathetic and parasympathetic nerves, for the most part the latter with prior evidence that loss of preganglionic parasympathetic system innervation decreased tear secretion., ${ }^{5,6}$ The sympathetic contribution seems to vary among different species and its role in promoting tearing is controversial, ${ }^{7}$ with electrostimulation of the superior cervical ganglion shown to increase tear secretion $^{8}$ or to be ineffective. ${ }^{9}$

To address the question of contribution, Jin et $\mathrm{al}^{4}$ denervated all postganglionic nerve bundles [postganglionic denervation (PGD)]. Basal tearing was decreased after denervation for up to seven days, demonstrating that the postganglionic nerves are responsible for basal tearing. Denervation also abolished reflex tearing, possibly due to loss of the reflex circuit. Subsequent continuous infusion of sympathetic system agonists norepinephrine, phenoxybenzamine, and propranolol did not alter tear secretion or lacrimal gland weight of normal glands after postganglionic denervation, revealing that the sympathetic system is not involved in either tear secretion or atrophy after postganglionic denervation surgery. On the other hand, infusion of the parasympathetic nicotinic and muscarinic acetylcholine receptor agonist carbachol increased tearing and reduced lacrimal gland atrophy after postganglionic denervation, suggesting that alterations of the lacrimal gland after denervation are due to depletion of parasympathetic neural input to the lacrimal gland, with little or no input of the sympathetic system. These results corroborate an extensive literature on this subject. ${ }^{5-7}$

\section{Postganglionic Denervation Result in Lacrimal Gland Atrophy}

Particularly interesting were the morphologic changes after denervation. As could be expected, loss of neural stimuli to the lacrimal gland resulted in lacrimal gland atrophy, massive inflammation, and fewer myoepithelial cells. Curiously, ischemia was not a main factor in atrophy. Instead, there was an abrupt change in the lacrimal gland

\footnotetext{
Accepted for publication March 2, 2020.

Disclosures: None declared.

Address correspondence to Karina Dias-Teixeira, Ph.D., PO Box 800732, Pinn Hall 3005, Charlottesville, VA 22908-0732. E-mail: kld5u@ virginia.edu
} 
energy status, with increasing reactive oxygen species (ROS) and decreasing adenosine triphosphate (ATP) generation as well as acinar cell mitochondrial damage. Also of interest was a decrease in phosphorylation of mechanistic target of rapamycin kinase (MTOR; mTOR) and of its substrate ribosomal protein S6 kinase B1 (RPS6KB1; S6K1), and increased nucleoporin 62 [NUP62; ubiquitin binding-protein 62 (p62)] and lipidated form of LC3 [microtubule-associated protein 1 light chain 3 (LC3II)], suggesting induction of autophagy. How autophagy might contribute to or counteract lacrimal gland atrophy needs further investigation. At an early stage, autophagy is characterized by activation of Unc-51 like autophagy activating kinase 1 (ULK1) in a complex with autophagy-related protein 3 (ATG3), focal adhesion kinase family integrating protein (FIP2000), and autophagy-related protein 101 (ATG101), resulting in autophagosome formation. ${ }^{10}$ How autophagy is triggered after denervation and the autophagyrelated proteins require more investigation, as well the stage of the autophagy process and participation of factors such as ATG3 and ATG101. The links between autophagy, ROS, ATP, and mitochondrial damage should also be explored in detail to better understand the oxidative damage to the acinar cells, and if some mechanism such as mitophagy is taking place after denervation to remove damaged and malfunctioning mitochondria.

\section{Perspectives}

Additional investigation on parasympathetic nerve regulation of the lacrimal gland is necessary, as well a deep understanding of the role of autophagy in this system. This information may shed light on new approaches to treating and even preventing dry eye disease.

\section{References}

1. Karnati R, Laurie DE, Laurie GW: Lacritin and the tear proteome as natural replacement therapy for dry eye. Exp Eye Res 2013, 117: $39-52$

2. Dartt DA, Willcox MDP: Complexity of the tear film: importance in homeostasis and dysfunction during disease. Exp Eye Res 2013, 117: $1-3$

3. Craig JP, Nichols KK, Akpek EK, Caffery B, Dua HS, Joo CK, Liu Z, Nelson JD, Nichols JJ, Tsubota K, Stapleton F: TFOS DEWS II definition and classification report. Ocul Surf 2017, 15:276-283

4. Jin K, Imada T, Hisamura R, Ito M, Tanaka FK, Nakamura S, Tsubota K: Identification of lacrimal gland postganglionic innervation and its regulation of tear secretion. Am J Pathol 2020, 190: 1068-1079

5. Dartt DA: Neural regulation of lacrimal gland secretory processes: relevance in dry eye diseases. Prog Retin Eye Res 2009, 28:155-177

6. Ding C, Walcott B, Keyser KT: Neuronal nitric oxide synthase and the autonomic innervation of the mouse lacrimal gland. Invest Ophthalmol Vis Sci 2001, 42:2789-2794

7. Ding C, Walcott B, Keyser KT: Sympathetic neural control of the mouse lacrimal gland. Invest Ophthalmol Vis Sci 2003, 44:1513-1520

8. Tangkrisanavinont V: Stimulation of lacrimal secretion by sympathetic nerve impulses in the rabbit. Life Sci 1984, 34:2365-2371

9. Botelho SY, Hisada M, Fuenmayor N: Functional innervation of the lacrimal gland in the cat. Origin of secretomotor fibers in the lacrimal nerve. Arch Ophthalmol 1966, 76:581-588

10. Kim J, Kundu M, Viollet B, Guan KL: AMPK and mTOR regulate autophagy through direct phosphorylation of Ulk1. Nat Cell Biol 2011, $13: 132-141$ 